\title{
Review of Feasibility Studies to Ensure Conducting the Proper Nursing Intervention Research
}

\author{
Michiyo Oka*, RN, PhD, Satsuki Takahashi, RN, MHS and Mitsuhiro Matsumoto, RN, MSN, CNS
}

Gunma University, Graduate School of Health Sciences, Japan

*Corresponding author: Michiyo Oka, RN, PhD, Gunma University, Graduate School of Health Sciences, 3-39-22 Showa, Maebashi, Gunma, 371-8514, Japan, Tel: +81-27-220-8819, Fax: +81-27-220-8926

\begin{abstract}
When introducing a new nursing system, it is the responsibility of the introducing side to incorporate what has been confirmed. However, it is difficult to conduct a large-scale randomized controlled trial (RCT) to confirm the evidence according to the protocol. Therefore, as a nursing researcher, we would like to utilize feasibility research. In this paper, we will introduce the necessity of feasibility study, the definition of feasibility study, and the parameters for high-quality feasibility study based on the literature.

The need for feasibility study is that it is necessary to confirm in advance whether main studies by large-scale RCTs can be carried out according to the protocol.

The definition of feasibility study is a study to confirm whether the main studies can be done as planned, and the pilot study is a part of the feasibility study. In addition, feasibility study can be divided into three types: 'randomised pilot studies', 'non-randomized pilot studies', and 'other feasibility studies'. Parameters for high-quality feasibility study are evaluated using not only changes in quantitative parameters such as blood test data, but also qualitative parameters such as interview results.

It can be said that conducting feasibility studies before the main study, confirming whether the evidence of nursing activities can be verified, and not giving up the verification of the evidence will lead to the development of nursing science.
\end{abstract}

\section{Keywords}

Feasibility study, Pilot study, Intervention studies

\section{Introduction}

Introducing a new nursing system such as a patient education program or a nursing human resource devel- opment system has the advantage of making the quality of nursing constant and sharing guidelines with the target people. When introducing a nursing system, it can be said that it is the responsibility of the introducing side to incorporate the system which effect has been confirmed.

Large-scale randomized controlled trials (RCTs) interventions are required to confirm the evidence, but it is difficult to immediately conduct large-scale intervention studies according to the exact protocol. In addition, many nursing interventions are aimed at psychosocial interventions such as alleviation of anxiety, and are often influenced by the willingness of clinical nurses to intervene.

Therefore, we recommend the use of feasibility studies. Feasibility studies can confirm the feasibility of nursing intervention studies and whether they can be conducted reliably. The purpose of this review is to introduce the need for feasibility study, the definition of feasibility study, and the parameters for quality feasibility study.

\section{Necessity of Feasibility Study}

RCTs are required to conduct high-evidence research. In addition, when writing a research paper, it may be required to write based on the reporting guidelines of Equator Network (Enhancing the QUAlity and Transparency Of health Research net work) [1], which contains guidelines for international research quality. Each item of this guideline is just a description item when writing a research, but in other words, it means that it is neces- 
sary to plan according to these items when conducting future research.

However, it is difficult to plan nursing intervention studies according to these guidelines. For example, in RCTs, it is desirable to plan to meet each item of CONSORT checklist 2010 [2], but in clinical studies such as patient education, complete concealment or blinding when grouping participants is extremely difficult. It is difficult to do. In 2018, the CONSORT-SPI 2018 Extension [3] was published to report on psychosocial intervention studies, changing from blinding to masking in the literature. However, even in that literature, the method of determining the number of participants is required to be described in the same way as CONSORT checklist 2010. In other words, even with the CONSORT-SPI 2018 Extension, it is necessary to recruit a certain number of participants, and it is a high hurdle.

When the author (MO) also conducted an RCT using a patient education program called the 'EASE Program' [4], the author (MO) spent a great deal of effort to ask a thirteen medical facility to meet the required number of participants. Also, regarding the stepped wedge cluster randomized trial (SW-CRT), Kristunas, et al. [5] said, 'without first testing the implementation of the intervention, it may be difficult to determine how long the intervention will take to embed in, and therefore how long the periods need to be between clusters starting the intervention'. Even in the RCT conducted by the authors mentioned above [4], before starting the main study, there are psychological concerns such as whether all facilities can be executed according to the protocol, and a great deal of time and financial effort is required.

When implementing nursing systems, evidence of the system should be proven before utilizing them. However, from the above, it can be seen that there are various hurdles to conducting research that meets the CONSORT checklist for proof of evidence. Not only that, but even if the evidence is proven, it is not always possible to provide a nursing system that is suitable for the site of intervention. Therefore, it can be said that it is necessary to confirm in advance by feasibility study whether main studies can be carried out according to the protocol by large-scale RCT. Funder may require the implementation of feasibility study before research with large research funds.

\section{What is Feasibility Study? -Differences from Pilot Research}

Feasibility studies are being conducted in many clinical fields and are increasing year by year. After filters applied 'nursing journals', if you enter 'feasibility study' in the search query in PubMed, 154 studies will be hit in 2009. The number of studies is increasing every year, with 344 studies in 2020, more than doubling in 10 years.
The definitions and explanations of feasibility studies are given by each researcher, and some definitions are shown in Table 1. In these definitions, when describing feasibility studies, differences from pilot studies are also taken up, and it is clear that it is necessary to distinguish between the two. For example, Everitt [6] and Thabane, et al. [7] state that feasibility study and pilot study are almost synonymous. However, Eldridge, et al. [8] argued that there was no distinction between feasibility studies and pilot studies. So they to develop the framework of both studies they undertook a Delphi survey; ran an open meeting at a trial methodology conference; conducted a review of definitions outside the health research context; consulted experts at an international consensus meeting; and reviewed 27 empirical pilot or feasibility studies [8]. As a result, they finally conclude that 'In our framework, pilot studies are a subset of feasibility studies, rather than the two being mutually exclusive'. Their explanation of 'pilot studies are a subset of feasibility studies', can be said to explain the difference between feasibility studies and pilot studies so far in a very easy-to-understand manner.

Eldridge, et al. [8] further illustrate the conceptual framework of feasibility and pilot studies (Figure 1). They state that 'The figure indicates that where there is uncertainty about future RCT feasibility, a feasibility study is appropriate'. The figure also shows that there are three types of feasibility studies.

The first type is randomised pilot studies, which states that 'Randomised pilot studies are those studies in which the future RCT, or parts of it, including the randomisation of participants, is conducted on a smaller scale (piloted) to see if it can be done. 'There is a description of 'internal pilot', which corresponds to 'some cases, this (pilot studies) will be the first phase of the main study, and data from the pilot phase may contribute to the final analysis; this can be referred to as an internal pilot' as defined by NIHR (National Institute for Health Research) [9].

The second type of feasibility study is 'non-randomized pilot studies'. Non-randomised pilot studies are 'similar to randomised pilot studies; they are studies in which all or part of the intervention to be evaluated and other processes to be undertaken in a future trial is/ are carried out (piloted) but without randomisation of participants. These could also legitimately be called by the umbrella term, feasibility study. These studies cover a wide range from those that are very similar to randomised pilot studies except that the intervention and control groups have not been randomised, to those in which only the intervention, and no other trial processes, are piloted'[8].

The third type of feasibility study is 'other feasibility studies'. This 'other feasibility studies' is described by Eldridge, et al. [8] as 'Feasibility studies that are not pilot studies are those in which investigators attempt to 


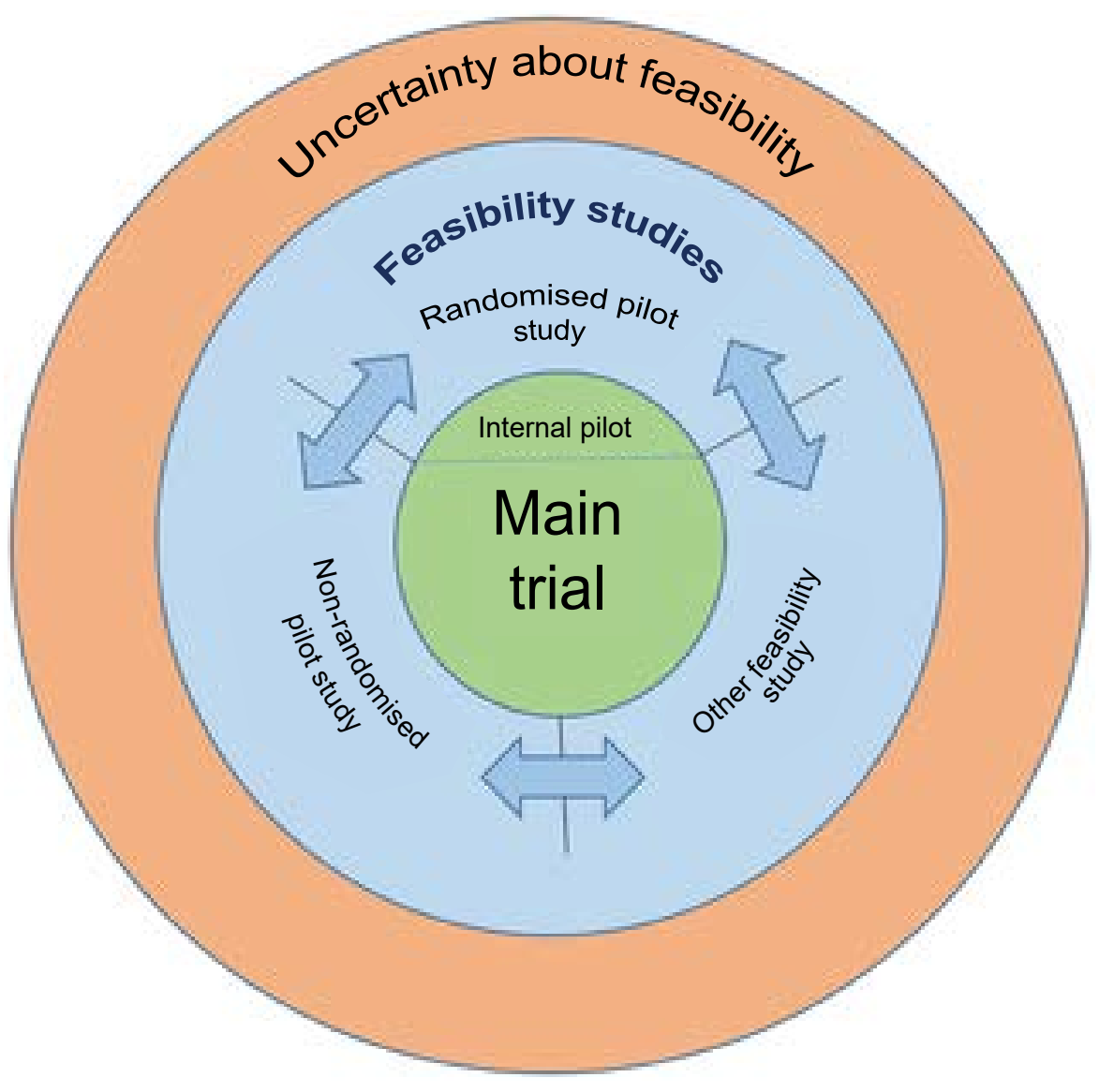

Figure 1: Conceptual framework of feasibility and pilot studies (Eldridge, et al. [8]).

Table 1: Definition of feasibility studies and pilot studies.

\begin{tabular}{|c|c|c|}
\hline & Definition of Feasibility Studies & Definition of Pilot Studies \\
\hline Everitt [6] & Essentially a synonym for pilot study. & $\begin{array}{l}\text { A small-scale investigation designed } \\
\text { either to test the feasibility of methods } \\
\text { and procedures for later use on a large } \\
\text { scale, or to search for possible effects } \\
\text { and associations that may be worth } \\
\text { following up in a subsequent larger } \\
\text { study. }\end{array}$ \\
\hline $\begin{array}{l}\text { Thabane, et } \\
\text { al. [7] }\end{array}$ & $\begin{array}{l}\text { Feasibility studies are routinely performed in many clinical areas. } \\
\text { It is fair to say that every major clinical trial had to start with some } \\
\text { piloting or a small scale investigation to assess the feasibility of } \\
\text { conducting a larger scale study }\end{array}$ & $\begin{array}{l}\text { 'A pilot study is synonymous with a } \\
\text { feasibility study intended to guide the } \\
\text { planning of a large scale investigation' }\end{array}$ \\
\hline $\begin{array}{l}\text { Eldridge, et } \\
\text { al. [8] }\end{array}$ & $\begin{array}{l}\text { 'In our framework, pilot studies are a subset of feasibility studies, } \\
\text { rather than the two being mutually exclusive'. } \\
\text { 'A feasibility study asks whether something can be done, should we } \\
\text { proceed with it, and if so, how'. }\end{array}$ & $\begin{array}{l}\text { A pilot study asks the same questions } \\
\text { but also has a specific design feature: } \\
\text { In a pilot study a future study, or part of } \\
\text { a future study, is conducted on a smaller } \\
\text { scale. }\end{array}$ \\
\hline NIHR [9] & $\begin{array}{l}\text { Feasibility studies are pieces of research done before a main study } \\
\text { in order to answer the question "Can this study be done?". They are } \\
\text { used to estimate important parameters that are needed to design } \\
\text { the main study. The design of a feasibility study generally involves } \\
\text { listing those parameters which are uncertain and describing the } \\
\text { methods for improving their precision so that the main study will } \\
\text { have a better chance of success'. } \\
\text { 'Feasibility studies do not evaluate the outcome of interest; that is } \\
\text { left to the main study. Feasibility studies for randomised controlled } \\
\text { trials may not themselves be randomised. If a feasibility study is } \\
\text { a small randomised controlled trial, it does not necessarily need } \\
\text { to have a primary outcome or power calculations. Instead, the } \\
\text { sample size is often used to estimate the critical parameters (e.g. } \\
\text { recruitment rate) to the necessary degree of precision'. }\end{array}$ & $\begin{array}{l}\text { 'Pilot studies are a version of the main } \\
\text { study that is run in miniature to test } \\
\text { whether the components of the main } \\
\text { study can work together. It is focused on } \\
\text { ensuring that the processes of the main } \\
\text { study (e.g. recruitment, randomisation, } \\
\text { treatment, and follow-up assessments) } \\
\text { all run smoothly. It will therefore } \\
\text { resemble the main study in many } \\
\text { respects, including an assessment of the } \\
\text { primary outcome. }\end{array}$ \\
\hline
\end{tabular}


answer a question about whether some element of the future trial can be done but do not implement the intervention to be evaluated or other processes to be undertaken in a future trial, though they may be addressing intervention development in some way'.

This figure clearly explains the relationship between feasibility studies and pilot studies, and also visually explains that there are three types of feasibility studies. NIHR [9] in the United Kingdom defines feasibility studies as shown in Table 1. The notable feature here is 'If a feasibility study is a small randomized controlled trial, it does not necessarily need to have a primary outcome or power calculations. Instead, the sample size is often used to estimate the critical parameters (eg recruitment rate) to the necessary degree of precision' [9].

In feasibility studies, we should not focus on outcome evaluation, but rather on confirming the effectiveness at the place of implementation, such as whether the participants who are really planned will gather when conducting large-scale main studies. In other words, feasibility study is a study conducted prior to main studies, and it is possible to confirm the feasibility of the study by conducting a research method called feasibility study to see if nursing intervention research can be carried out reliably. Also noteworthy in the definition of pilot study is that it states 'Pilot studies are a version of the main study that is run in miniature to test'. Therefore, it is expected that the outcome evaluation will be performed in the pilot study and the result of the main studies will be estimated.

The above definitions can be summarized as follows:

- In the 2000s, the difference between feasibility studies and pilot studies was not clarified, but in the 2010s, the difference became clear.

- Feasibility studies are studies that are conducted prior to main studies, and are studies to confirm whether main studies can be performed as planned, and outcome evaluation is not always necessary.

- The pilot study is a miniature version of the study on whether or not the main studies can be carried out according to the protocol, and an outcome evaluation is conducted.
- Feasibility studies can be divided into three types: 'randomised pilot studies', 'Non-randomized pilot studies', and 'other feasibility studies'.

- Pilot research is part of feasibility research.

\section{Evaluation of High-Quality Feasibility Study}

What is a high quality feasibility study? In the feasibility study, we will simulate whether the main studies can be done according to the protocol. At that time, we confirmed some studies on the parameters to be evaluated in the feasibility study.

The CONSORT statement announces 'CONSORT 2010 statement: Extension to randomized pilot and feasibility trials' for pilot study and feasibility study [10]. The main change in The original CONSORT statement items and this an extension to the CONSORT statement for randomized pilot and feasibility trials is to specify in the 'title' and 'trail design' that it is a randomized pilot or feasibility trial. It is also to describe how participants were identified and consent obtained. In addition, it is required to describe their implications for the future definitive trial in 'conclusions'.

It is said to be this extension does not directly apply to internal pilot studies built into the design of a main trial, non-randomized pilot and feasibility studies, or phase II studies. This means that it does not apply to most of the figures in the Conceptual framework of feasibility and pilot studies by Eldridge, et al. [8]. However, these studies shown in 'CONSORT 2010 statement: Extension to randomised pilot and feasibility trials' are all have some similarities to randomized pilot and feasibility studies and so many of the principles might also apply.

In NIHR [9], which was also introduced in the definition section, the contents of Table 2 are introduced as an example of feasibility study parameters. The characteristic of the above parameters is that they include not only quantitative indicators but also qualitative indicators. For example, 'Number of eligible patients, carers or other appropriate participants' is given as a parameter. This is a quantitative parameter. However, parameters such as 'Willingness of participants to be randomized' and 'Willingness of clinicians to recruit participants' are

Table 2: Example of feasibility study parameters (NIHR [9]).

- Standard deviation of the outcome measure, which is needed in some cases to estimate sample size

- Willingness of participants to be randomised

- Willingness of clinicians to recruit participants

- Number of eligible patients, carers or other appropriate participants

- Characteristics of the proposed outcome measure and in some cases feasibility studies might involve designing a suitable outcome measure

- Follow-up rates, response rates to questionnaires, adherence/compliance rates, ICCs in cluster trials, etc.

- Availability of data needed or the usefulness and limitations of a particular database

- Time needed to collect and analyse data 
also listed. These are qualitative parameters. If you collect information by qualitative methods such as participating in observations and interviewing the movements of nurses in the field, you will find improvements for the main study.

For example, among nursing research, Wade, et al. [11] research is an example of using feasibility study. The aim of this study is to develop and test the feasibility of a psychological intervention to reduce acute stress and prevent future morbidity. In this study, for patient with acute stress, to deliver stress support sessions and a relaxation and recovery program to them. As an evaluation, Wade uses nurse-reported qualitative patient feedback. This is exactly the qualitative parameter. As the patient feedback, there was an opinion that "Some were disappointed not to find calming classical music on DVD". For the relaxation and recovery program. Therefore, the researchers have added calming classical music tracks were added to the DVD as 'Refinement of the intervention post feasibility study'. It can be said that this uses the qualitative parameter as the evaluation of feasibility.

In this way, the feasibility study uses parameters related to quality as well as quantity to simulate whether main studies can be performed according to the protocol. This is a qualitative and careful confirmation that cannot be measured by objective evaluation alone, and leads to gaining valuable suggestions for the main study in nursing interventions that often intervene in psychosocial factors.

\section{Conclusion}

In this manuscript, we have described the necessity of feasibility study, the definition of feasibility study, and the parameters for high-quality feasibility study.

When conducting psychosocial interventions, it is difficult to make the conditions of the participants the same, and it may not be possible to know whether a large-scale RCT can follow the protocol.

Sometimes we don't have the time or money to gather people, or we don't have a clear prediction of primary outcomes, so we may want to give up on a large RCT. In such a case, by performing feasibility study first, it is possible to confirm whether the protocol seems to be as planned and where there is an improvement. It can be said that conducting feasibility studies before the main study will lead to the development of nursing science by not giving up the verification of evidence of nursing activities.

\section{Sources of Support}

This work was supported by JSPS KAKENHI Grant Number JP 20K10707.

\section{Conflict of Interest}

There are no conflicts of interest to declare.

\section{References}

1. Equator (Enhancing the QUAlity and Transparency Of health Research) network.

2. Schulz KF, Altman DG, Moher D, CONSORT Group (2010) CONSORT 2010 statement: Updated guidelines for reporting parallel group randomised trials. BMC Med 8: 18.

3. Montgomery P, Grant S, Mayo-Wilson E, Macdonald G, Michie S, et al. (2018) Reporting randomised trials of social and psychological interventions: The CONSORT-SPI 2018 Extension. Trials 19: 407.

4. Joboshi H, Oka M (2017) Effectiveness of an educational intervention (the Encourage Autonomous Self-Enrichment Program) in patients with chronic kidney disease: A randomized controlled trial. Int J Nurs Stud 67: 51-58.

5. Kristunas CA, Hemming K, Eborall H, Eldridge S, Gray LJ (2019) The current use of feasibility studies in the assessment of feasibility for stepped-wedge cluster randomised trials: A systematic review. BMC Med Res Methodol 19: 12.

6. Everitt BS (2006) Medical statistics from A to Z: A guide for clinicians and medical students. ( $2^{\text {nd }}$ edn), Cambridge University Press, Cambridge, 93-176.

7. Thabane L, Ma J, Chu R, Cheng J, Ismaila A, et al. (2010) A tutorial on pilot studies: The what, why and how. BMC Med Res Methodol 10: 1.

8. Eldridge SM, Lancaster GA, Campbell MJ, Thabane L, Hopewell S, et al. (2016) Defining feasibility and pilot studies in preparation for randomised controlled trials: Development of a conceptual framework. PLoS One 11: e0150205.

9. National Institute for Health Research (2019) Guidance on applying for feasibility studies.

10. Eldridge SM, Chan CL, Campbell MJ, Bond CM, Hopewell S, et al. (2016) CONSORT 2010 statement: Extension to randomised pilot and feasibility trials. Pilot Feasibility Stud 2: 64 .

11. Wade D, Als N, Bell V, Brewin C, D’Antoni D, et al. (2018) Providing psychological support to people in intensive care: Development and feasibility study of a nurse-led intervention to prevent acute stress and long-term morbidity. BMJ Open 8: e021083. 